\title{
A comparison of three clustering methods for finding subgroups in MRI, SMS or clinical data: SPSS TwoStep Cluster analysis, Latent Gold and SNOB
}

Peter Kent ${ }^{1 *}$, Rikke K Jensen ${ }^{2}$ and Alice Kongsted ${ }^{1,3}$

\begin{abstract}
Background: There are various methodological approaches to identifying clinically important subgroups and one method is to identify clusters of characteristics that differentiate people in cross-sectional and/or longitudinal data using Cluster Analysis (CA) or Latent Class Analysis (LCA). There is a scarcity of head-to-head comparisons that can inform the choice of which clustering method might be suitable for particular clinical datasets and research questions. Therefore, the aim of this study was to perform a head-to-head comparison of three commonly available methods (SPSS TwoStep CA, Latent Gold LCA and SNOB LCA).

Methods: The performance of these three methods was compared: (i) quantitatively using the number of subgroups detected, the classification probability of individuals into subgroups, the reproducibility of results, and (ii) qualitatively using subjective judgments about each program's ease of use and interpretability of the presentation of results. We analysed five real datasets of varying complexity in a secondary analysis of data from other research projects. Three datasets contained only MRI findings ( $n=2,060$ to 20,810 vertebral disc levels), one dataset contained only pain intensity data collected for 52 weeks by text (SMS) messaging ( $n=1,121$ people), and the last dataset contained a range of clinical variables measured in low back pain patients ( $n=543$ people). Four artificial datasets $(n=1,000$ each) containing subgroups of varying complexity were also analysed testing the ability of these clustering methods to detect subgroups and correctly classify individuals when subgroup membership was known.

Results: The results from the real clinical datasets indicated that the number of subgroups detected varied, the certainty of classifying individuals into those subgroups varied, the findings had perfect reproducibility, some programs were easier to use and the interpretability of the presentation of their findings also varied. The results from the artificial datasets indicated that all three clustering methods showed a near-perfect ability to detect known subgroups and correctly classify individuals into those subgroups.
\end{abstract}

Conclusions: Our subjective judgement was that Latent Gold offered the best balance of sensitivity to subgroups, ease of use and presentation of results with these datasets but we recognise that different clustering methods may suit other types of data and clinical research questions.

Keywords: Cluster analysis, Latent Class Analysis, Head-to-head comparison, Reproducibility, MRI, SMS

\footnotetext{
* Correspondence: pkent@health.sdu.dk

${ }^{1}$ School of Sports Science and Clinical Biomechanics, University of Southern

Denmark, Campusvej 55, Odense M 5230, Denmark

Full list of author information is available at the end of the article
} 


\section{Background}

There is increasing interest in the identification of clinically important patient subgroups in order to better target treatment, make more accurate estimates of prognosis, and improve health system efficiency by providing the right treatment to the right patient at the right time $[1,2]$. This is especially so in non-specific health conditions that are highly prevalent, costly and have a high burden of disease. For example, most back pain is non-specific and yet it is the leading cause of disability globally [3]. Identifying subgroups of findings can also be useful in imaging data, such as Magnetic Resonance Imaging (MRI) findings [4,5] and longitudinal data describing clinical or life course trajectories [6]. Longitudinal data may be collected using many methods but an increasingly used method is via Short Message Service (SMS) text messaging [7].

There are various methodological approaches to identifying subgroups, although the same validation stages are required before clinical importance can be established $[8,9]$. Some statistical approaches to subgrouping work backwards from an outcome, such as using good response to a treatment, as a way to identify the clinical characteristics of people most likely to respond to that therapy [10]. Other statistical methods seek to identify clusters of symptoms and signs that differentiate people, in cross-sectional and/or longitudinal data. This approach was taken by Beneciuk et al. [11], who used cluster analysis of baseline fear avoidance data from patients in a clinical trial and found three distinct subgroups (low risk, high specific fear, and high fear and catastrophising) that were associated with different clinical trajectories.

Historically, cluster analysis methods (hierarchical or $\mathrm{k}$-means clustering) have been used but more recently these have been complemented by probabilistic (Bayesian) methods, such as Latent Class Analysis (LCA). Traditional cluster analysis methods initially create a distance measure of dissimilarity between individuals (such as a Euclidean distance), and then seek to determine the underlying subgroup structure by optimising the within-subgroup variability of individuals' distance measures and maximising their between group variability. In contrast, LCA methods initially use a probabilistic modeling approach (such as finite mixture modeling) to identify the likely distributions with the data and the likely placement of individuals within those distributions. They then seek to determine the optimal subgroup structure that explains the most variance while requiring the simplest specification of the model (the optimal balance between the most explanatory models and parsimonious models). In this study we use the term 'clustering methods' as an umbrella term to cover both 'distance-based cluster analysis' approaches and 'probability-based LCA' approaches.
LCA has a number of advantages, including being able: to better manage variables of mixed measurement types (dichotomous, ordinal, interval scales and scales of varying width), to better handle missing data, to provide classification probabilities for individual classification, to provide model-based parameters that can be used to classify new individuals not in the derivation sample, and to have greater classification accuracy [12-14]. LCA methods are now easily accessible to clinical researchers and the use of these computationally-intense software programs has been facilitated by the speed of contemporary computers.

There are many computer programs available for LCA but there is a scarcity of head-to-head comparisons published that can inform the choice of which LCA might be suitable for particular clinical datasets and research questions. There are only two such studies that we are aware of. Haughton et al. [15] compared three LCA programs (Latent Gold, poLCA and MCLUST) using a single dataset. All three programs identified the same number of subgroups, though there was some variation in the individuals allocated to those subgroups. Those authors indicated that their results may not hold for other datasets and that the use of poLCA and MCLUST require proficiency in the $\mathrm{R}$ programming environment and language. Bacher et al. [12] compared a distance-based cluster method (SPSS TwoStep), and two LCA methods (Latent Gold and ALMO) using five artificial datasets with known subgroups. TwoStep is a hybrid approach that uses a distance measure to separate individuals but uses a similar method to LCA to choose the optimal subgroup model, and it has been shown to perform consistently better than traditional hierarchical cluster techniques [13]. Bacher et al. found that TwoStep was least able and Latent Gold most able to detect the correct number of subgroups. In particular, TwoStep had difficulties when the dataset contained a mix of nominal and interval data.

However, there are other LCA methods readily available, other measures of technical performance and other more user-focused aspects for comparison. Furthermore, the performance of clustering methods can vary depending on the type of data being analysed [13] and most previous comparison studies have been written for a statistical audience rather than for clinical researchers.

Therefore the aim of this study was to perform, using a variety of types of clinical and artificial datasets, a headto-head comparison of three commonly available clustering methods (TwoStep, Latent Gold and SNOB), based on the evaluation criteria of: the number of subgroups detected, the classification probability of individuals to those subgroups, the reproducibility of the findings, and each computer program's ease of use and interpretability of the presentation of results. These evaluation criteria were orientated towards informing the decisions of clinical researchers, rather than statisticians, and therefore clinical 
rather than mathematical language is used and clinical considerations are emphasised.

\section{Method}

\section{Clustering software}

This study investigated the use of three clustering methods, each implemented within a separate software program: (i) TwoStep Cluster Analysis in IBM SPSS (version 19, SPSS Statistics/IBM Corp, Chicago IL, USA), which is available in the base package of this program (TwoStep) [16], (ii) Latent Class Modeling in Latent Gold (version 4.5, Statistical Innovations, Belmont MA, USA), which is the simplest of three LCA approaches available in this program (Latent Gold) [17], and (iii) 'vanilla' SNOB (version 1.15, Monash University, Melbourne, Australia), which is the most straightforward form of this program (SNOB) [18-20]. SNOB is playfully named for its ability to detect classes (subgroups) and uses the Minimum Message Length principle and finite mixture modeling to probabilistically identify latent classes.

These three clustering methods were tested using their software default settings. In the case of TwoStep, this was a log-likelihood distance measure. Clustering methods, when in exploratory mode, require some form of 'stopping rule' to allow determination of the optimal number of subgroups. LCA methods typically include rules designed to find the subgroup solution that explains the most variance while requiring the simplest specification of the model. Examples of such rules are the Schwarz's Bayesian Information Criterion (BIC), Akaike's Information Criterion, and Minimum Message Length principle. In the case of TwoStep, there is a choice of BIC or Akaike's Information Criterion, with a default setting of BIC, and the program automatically determines the optimal solution based on the chosen criterion. Latent Gold requires the analyst to choose the optimal model and provides a number of criteria that can be used to inform that choice, the most commonly used single criterion being BIC. When using Latent Gold, we increased the number of investigated clusters until BIC did not decrease any further and chose the subgroup model with the lowest BIC and fewest subgroups. SNOB uses only Minimum Message Length and fully automates the choice of model.

\section{Real data sets}

We analysed five datasets of varying size, type and complexity. All were a secondary analysis of real data collected for other research projects. Three datasets $\left(\mathrm{MRI}^{1}, \mathrm{MRI}^{2}, \mathrm{MRI}^{3}\right)$ contained only MRI findings (dichotomous scales), one dataset (SMS) contained only pain intensity data (0 to 10 interval scale) collected every week over a one-year period by SMS messaging, and the last dataset (clinical) contained a range of clinical variables (dichotomous, ordinal and continuous scales) measured in low back pain (LBP) patients. These datasets were purposefully chosen from those available in our research group to investigate whether the performance of these clustering programs was consistent across data size, type and complexity, as these characteristics can affect cluster models [12]. Permission was obtained from the custodians of each of these datasets for secondary use of the data within this project (Per Kjær, Rikke Kruger Jensen, Hanne Albert/Peter Kent, Alice Kongsted, Alice Kongsted, respectively).

Both Latent Gold and SNOB are able to model data in dichotomous, ordinal and continuous scales, whereas TwoStep can only model dichotomous and interval data [12]. Therefore, to be able to model data across all three clustering methods, variables in the MRI datasets that were originally coded in ordinal scales were recoded into dichotomous scales using arbitrary but clinically intuitive cut-points. The mixed data types in the clinical dataset were retained in their original formats to preserve the complexity of these data but this restricted our comparison of these data to results from Latent Gold and SNOB. An overview of the characteristics of the five data sets is presented in Table 1.

All three MRI datasets were analysed at an individual vertebral disc level, where each person in the study contributed five lumbar vertebral disc levels. The MRI ${ }^{1}$ dataset was collected as part of the Danish Backs on Funen' longitudinal cohort study, and was taken from the baseline cohort measurement that included a lumbar MRI ( $n=412$ people, 2,060 disc levels). Full details of the data collection and coding have been previously reported [21]. Briefly, this cohort of people was a representative sample of the Danish general population and, who as part of the data collected in the study, had MRIs. The MRI images were quantitatively coded by an experienced musculoskeletal research radiologist using a detailed and standardised research MRI evaluation protocol that has demonstrated high reproducibility [22].

The MRI ${ }^{2}$ dataset is from a cohort of patients $(n=631$ patients, 3,155 disc levels) who were potential participants in a randomised controlled trial [23]. The details of the data collection and coding have also been previously reported [4]. In summary, all participants were patients who had attended a Danish outpatient hospital department (the Spine Centre of Southern Denmark) from June 2006 to June 2008, where they had been referred from the primary care sector for a multidisciplinary evaluation. Potential participants were people who had LBP or leg pain of at least 3 on an 11-point Numerical Rating Scale, a duration of current symptoms from 2 to 12 months, were above 18 years of age, and who had received a lumbar MRI. The MRI images were quantitatively coded by the same research radiologist using 
Table 1 Characteristics of real datasets

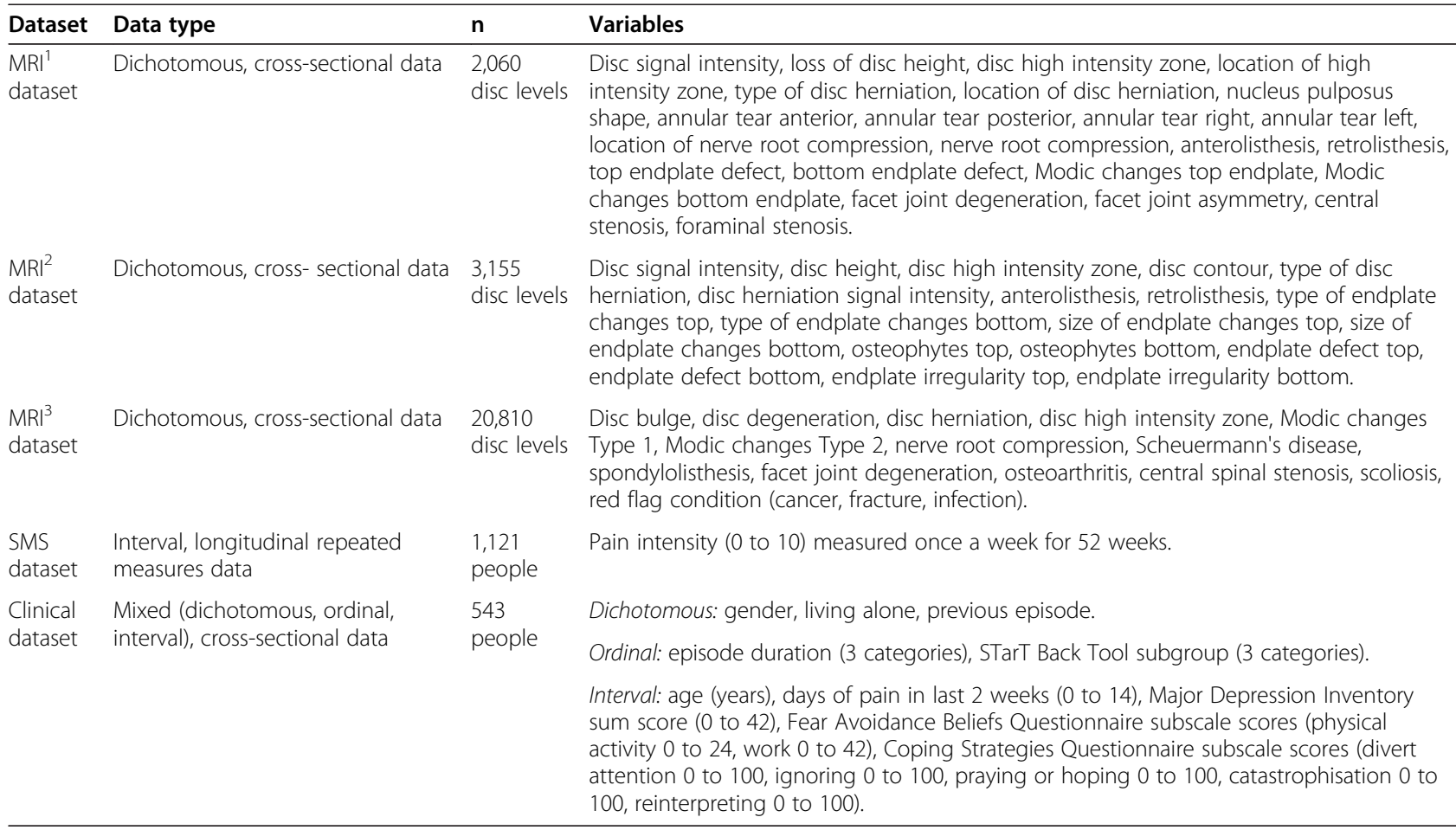

the same MRI evaluation protocol as in the $M R I^{1}$ dataset.

The $\mathrm{MRI}^{3}$ dataset was collected for a study on the prevalence of MRI-defined spinal pathologies [24] and a study of the reproducibility of coding MRI findings [25]. Full details of the data collection and coding have been reported in those studies but briefly, these data were extracted by three trained coders from the MRI reports of all people who had attended the outpatient medical department of the Spine Centre of Southern Denmark over an eight-year period (2000 to 2008) and received a lumbar spine MRI for which a narrative report could be retrieved from their electronic patient record $(n=4,162$ people, 20,810 disc levels). Once trained, the inter-rater reproducibility across the 14 pathoanatomic categories for a sample of these data ( $\mathrm{n}=1,700$ ratings) ranged from substantial to perfect [25]. The original MRI reports had been narrated by either of two experienced musculoskeletal radiologists.

The SMS dataset contained data on LBP intensity self-reported every week for one year by 1,121 primary care chiropractic or GP patients in Denmark. These data were collected as part of a currently unpublished cohort study designed to identify course patterns, subgroups and prognostic factors in LBP patients seeking care from general practitioners (GPs) and chiropractors. All GPs in the administrative region of Southern Denmark were invited to participate in a quality assurance program focusing on patients with LBP and the patient self-reported data used in the current study were recorded at or after the first consultation. The chiropractors were participants in a research collaboration with a clinical practice research unit that has previously been described [26]. Patient inclusion criteria were being aged 18-65 years, attending the GP or chiropractor for the first time due to the current episode of LBP, and having adequate Danish language competency. Exclusion criteria were a suspicion of inflammatory or pathological pain, and nerve root involvement requiring acute referral to surgery. The 52 weeks of pain intensity scores had a mean within-subject correlation (collinearity) over time of 0.59 (SD 0.11, full range 0.22 to 0.81). The SMS data were entered into the clustering models without reference to their time sequence, a method previously described [27].

The clinical dataset consisted of responses on an array of questionnaires from 543 people who were potential participants in a cross-sectional study of the STarT Back Screening Tool [28]. Full details of this data collection and coding have also been reported. Participants were primary care patients in 19 chiropractic clinics who were members of the same clinical practice research unit involved in the SMS dataset. Inclusion criteria were consenting people seeking care for LBP with adequate Danish literacy to understand and self-complete the questionnaire pack.

In all three clustering programs, all the variables from each dataset were simultaneously entered into the model as indicators, with no dependent, covariate or predictor variables specified. The data collection and analysis of 
each of the five datasets was performed with the approval of the scientific ethics committee appropriate for each study. Under Danish law, the secondary analysis of such de-identified data does not require separate ethics approval (The Act on Processing of Personal Data, December 2012, Section 5.2; Act on Research Ethics Review of Health Research Projects, October 2013, Section 14.2).

\section{Artificial data sets}

Four artificial datasets $(\mathrm{n}=1,000$ each) containing subgroups of varying complexity were created to test the ability of the clustering methods to detect subgroups and correctly classify individuals when subgroup membership was known to the researchers but withheld from the modeling process. The subgroup characteristics are described in Table 2 and illustrated in Figures 1, 2, 3 and 4. The variables were arbitrarily given clinical labels to aid comprehension but these labels were entirely fictitious.

To allow comparison across all three clustering methods, including TwoStep, these artificial datasets contained only interval $+/$ - dichotomous data. Each dataset contained nine variables that differentiated three to six subgroups based on their scoring pattern. The complexity of the range of scores that differentiated the subgroups varied from easy (discrete and mutually exclusive scoring bands) to more difficult (overlapping scoring bands plus the presence of 10 'pure noise' variables). Within each scoring band, the scores on each variable were calculated using random number generation (Excel for Mac 2008, Microsoft Corporation, Redmond, WA, USA). The sequence of individuals in the artificial datasets was randomised prior to analysis.

\section{Comparison criteria}

The performance of the three clustering methods was compared: (i) quantitatively using the number of subgroups detected, the classification probability of individuals into subgroups and the reproducibility of results, and (ii) qualitatively using subjective judgements about each computer program's ease of use and the ease of interpretation of the presentation of results.
The number of subgroups detected by each method was reported, along with a summary of the classification probability of each individual disc level or patient. The classification probability is an index of the certainty with which each individual was allocated into a subgroup based on their scoring pattern. For example, individuals with a scoring pattern that is stereotypical of a subgroup will be allocated with more certainty than individuals whose scoring pattern is on the boundary between two subgroups. Classification probability of individuals was not available in the TwoStep procedure.

Furthermore, the reproducibility of each method's findings was measured by performing 10 repetitions of the clustering for each dataset. Reproducibility was reported using the number of subgroups detected, classification stability (agreement on which subgroup each individual disc-level or patient was allocated to), and classification probability (certainty of the subgroup allocation of each individual). Descriptive statistics (proportions, means, standard deviations (SD) or 95\% confidence intervals $(95 \% \mathrm{CI})$ ) and trends in the number of subgroups detected were reported. Differences between classification confidence were tested using the STATA prtesti command for a one-sample test of proportions (Stata Corp, College Station, Texus, USA).

Pair-wise classification disagreement between clustering methods on the allocation of individuals into subgroups was also calculated. Subgroup membership of all individuals was cross-tabulated between the final subgroup models from each clustering method, allocating each individual to the cluster in which they had the highest posterior probability. The subgroups with the highest number of individuals in these cross-tabulations were deemed to be the same subgroup and individuals classified by one method but not the other as being in that subgroup were deemed to be disagreements. The total number of pair-wise disagreements at an individual level was expressed as a proportion of the total sample size. In the case of SNOB, this process was facilitated by a tree diagram showing the derivation of the subgroups in the final model. The tree is based on a type of Bhattacharyya Coefficient that measures the similarity among subgroup probability distributions. A visual example of how this cross-tabulation was performed is shown in Figure 5.

Table 2 Characteristics of artificial datasets

\begin{tabular}{cccc}
\hline Dataset & No of subgroups & Data type & Subgroup scoring \\
\hline A1 & 3 & Interval and dichotomous & Discrete scoring bands \\
A2 & 3 & Interval & Overlapping scoring bands \\
A3 & 6 & Interval and dichotomous & $\begin{array}{c}\text { Overlapping scoring bands with two } \\
\text { distinct subgroups on each variable } \\
\text { A4 }\end{array}$ \\
& 3 & Interval & Overlapping scoring bands plus 10 'noise' \\
variables that do not discriminate subgroups
\end{tabular}




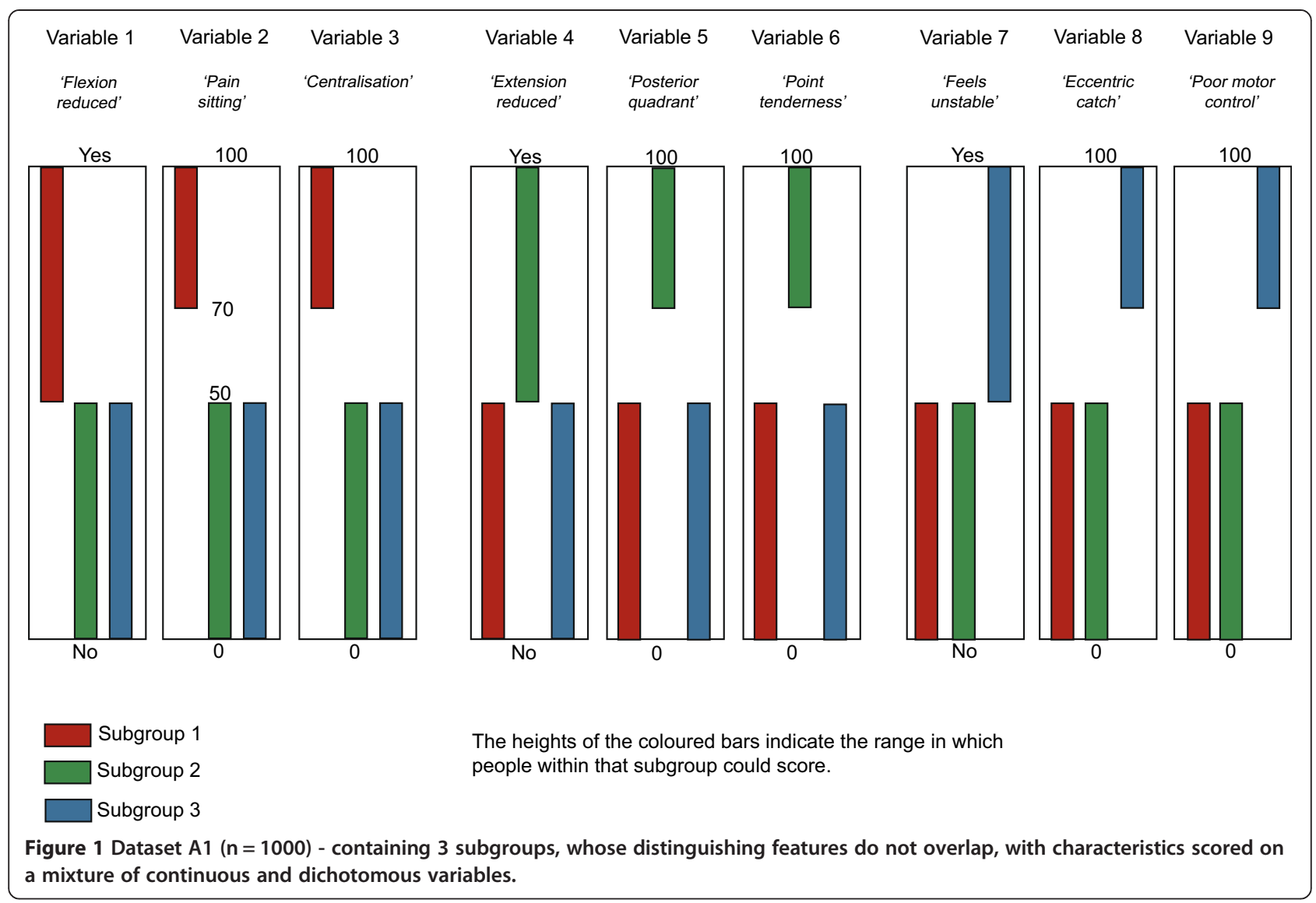

\section{Results}

\section{Real datasets}

As shown in Table 3, the number of subgroups detected by each of the clustering methods varied. In every dataset, TwoStep detected the least number of subgroups, Latent Gold detected more subgroups and SNOB detected the most. This indicates that the clustering methods varied in their sensitivity to scoring patterns within the same dataset. The differences in the number of subgroups detected were typically smaller between Latent Gold and SNOB than between either of these and TwoStep, although the SMS dataset was an exception to this observation. This may have been due to a differential effect resulting from the amount of collinearity in these data, as independence of the included variables is a common assumption in clustering methods.

Classification certainty (probability) was not available for TwoStep but is displayed at a group-average level in Table 3 for Latent Gold and SNOB. The standard deviation (SD) is also displayed and gives an index of the classification uncertainty that those clustering methods had in allocating individuals to subgroups. The classification certainty did not differ between Latent Gold and SNOB in the MRI ${ }^{1}$ dataset $(p=0.625)$ or the clinical dataset $(p=0.246)$, but it did differ in the $\mathrm{MRI}^{2}$ and
$\mathrm{MRI}^{3}$ datasets and the SMS dataset (all $\mathrm{p}<0.001$ ). However, despite an expectation that the clustering method that was most sensitive to subgroup differences (SNOB) would also be the most certain, this was not consistently observed, as the average classification certainty was not always higher for SNOB and the differences between the methods were typically small.

The between-clustering method classification disagreement of individuals (disc levels or patients) is shown in Figure 6. The pairwise classification disagreement varied between comparisons of clustering methods, as seen by the non-overlapping confidence intervals, but there was no consistent trend that would have indicated that across datasets, some of the clustering methods more often agreed with each other.

The results for reproducibility (number of subgroups, allocation to subgroups, classification probability) are also shown in Table 3. These tests of the consistency of findings within each clustering program during 10 replications of the analysis of each dataset showed $100 \%$ agreement in all datasets and on all types of test (number of subgroups, allocation to subgroups, classification probability). In the case of Latent Gold, the default setting is to commence each analysis with a random seed point, which predictably results in some 


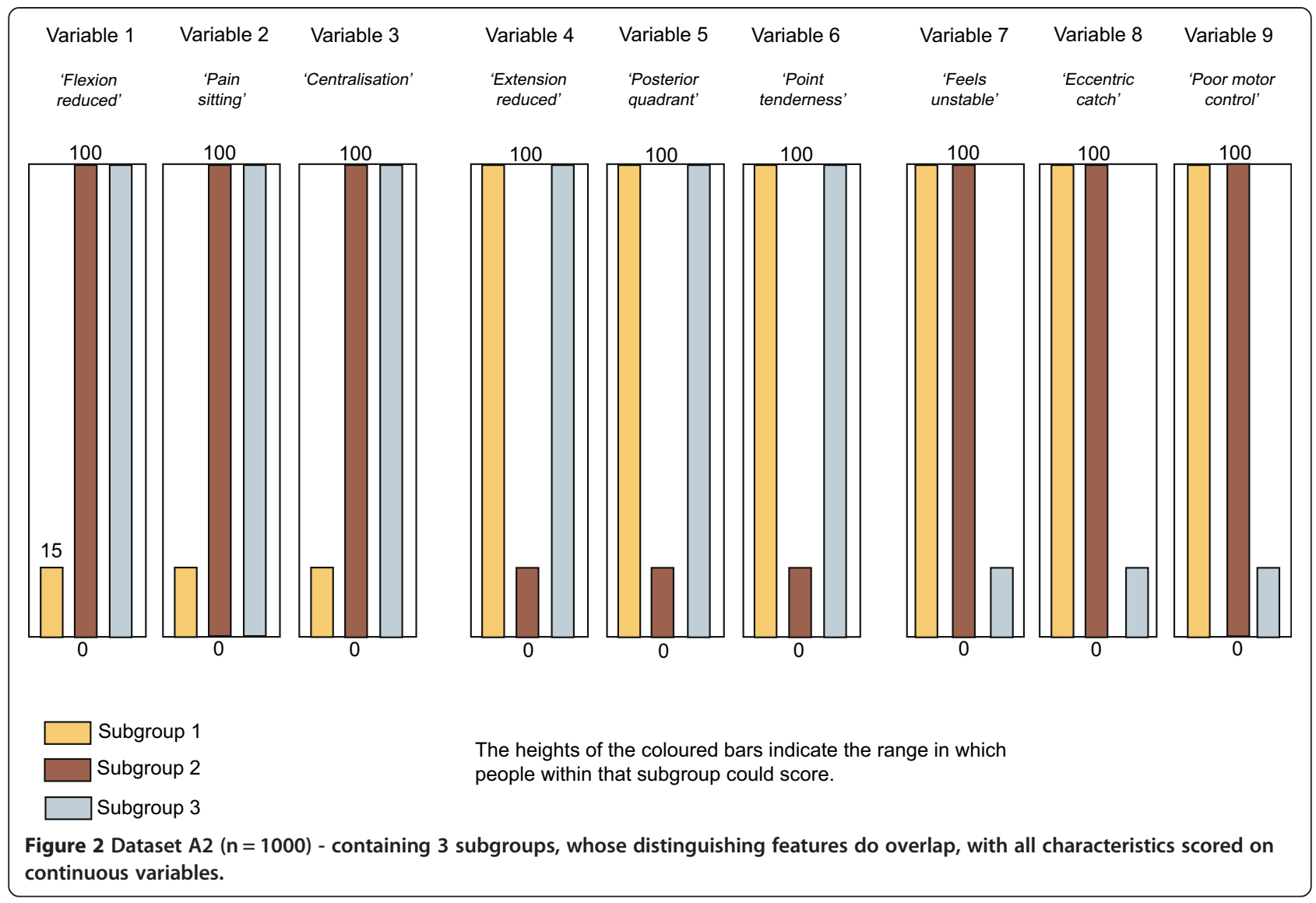

variability of the findings. As the other two clustering programs commence with a fixed but arbitrary seed point, to standardise these comparisons of reproducibility, we used a fixed but arbitrary seed point in Latent Gold in this part of the analysis.

\section{Artificial datasets}

As shown in Table 4, these three clustering methods displayed a near-perfect ability to detect known subgroups. The only exception was that Latent Gold split one subgroup into two in artificial dataset 3 that was

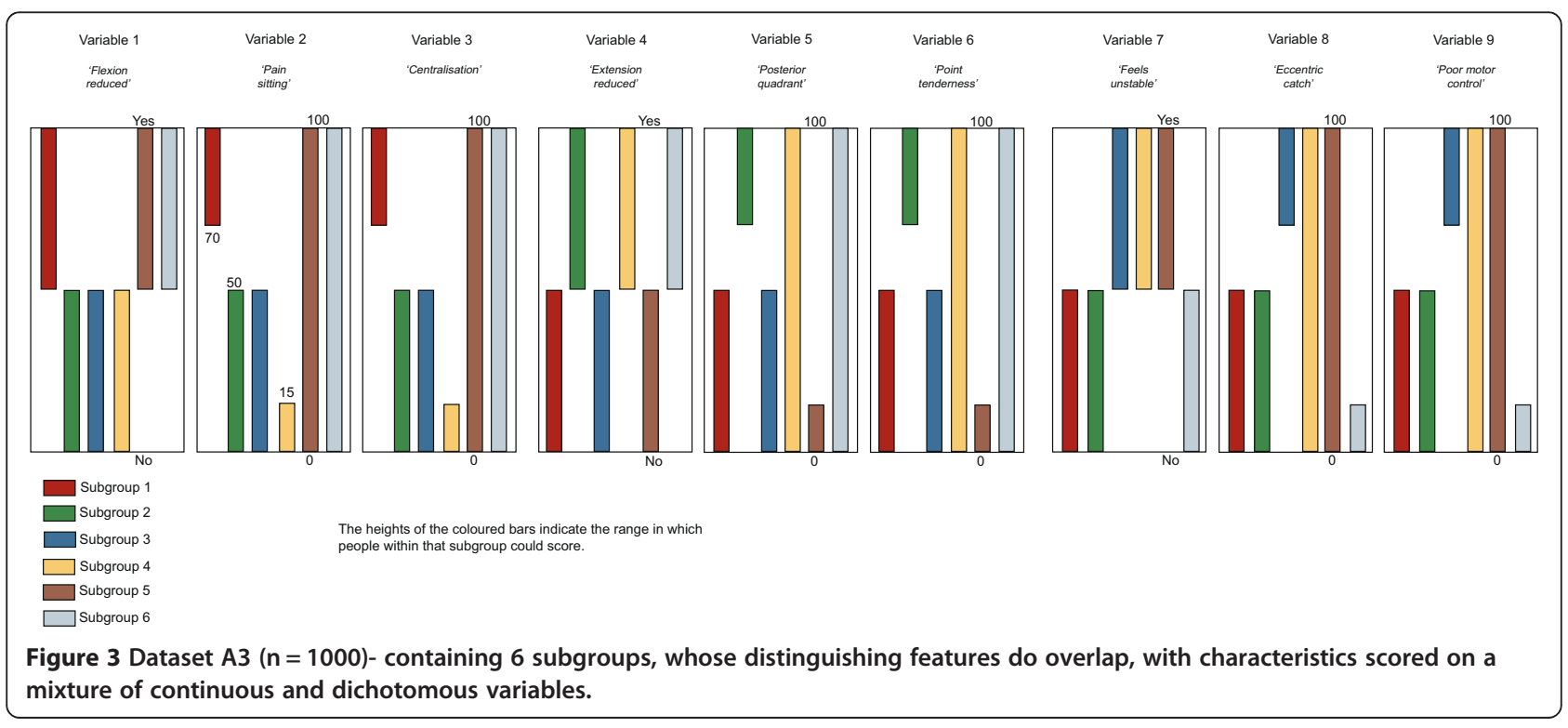




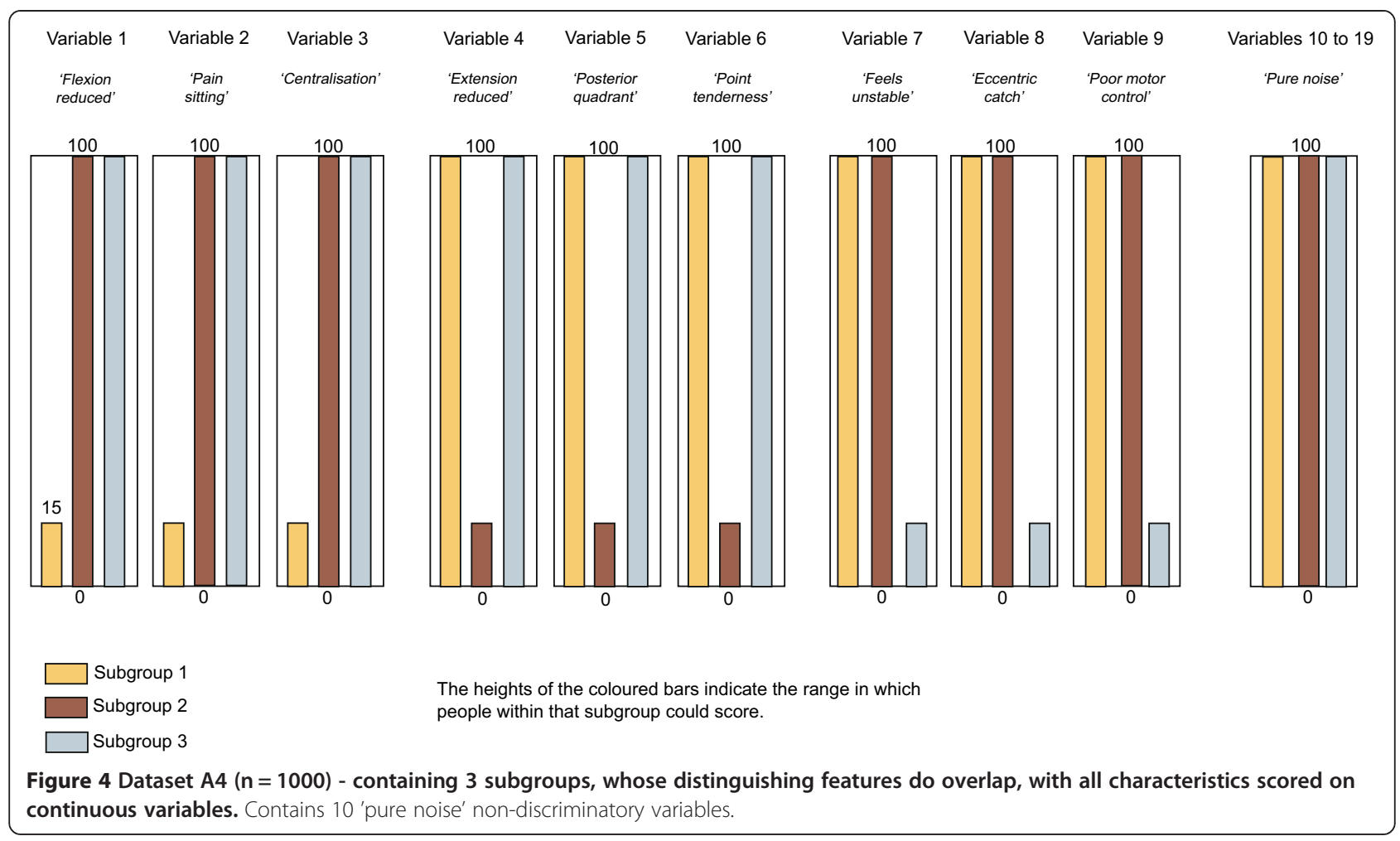

designed to contain 6 subgroups. We cannot rule out that our method of using random number generation to produce individual scores had produced a scoring characteristic that Latent Gold detected and used as the basis for splitting a 'mother subgroup' into two 'daughter subgroups'. The classification accuracy was also very high, ranging from $98.4 \%$ to $100 \%$.

\section{Ease of use, interpretability, cost}

Our subjective judgement is that these three clustering programs also varied in their ease of use and the interpretability of their presentation of results. TwoStep has the easiest learning curve, with software commands that can be all menu-driven, there is plain-language explanatory material available via the internet, the optimal subgroup solution is automatically determined, and the results are presented numerically and graphically (charts of certainty of the subgroup structure, bar and pie charts of cluster frequencies, and charts displaying the importance of specific variables to subgroups). A limitation is that Two Step is not designed to analyse ordinal data and while it is technically possible to handle such data via the use of dummy variables, this disproportionally loads the distance measure on that variable with unpredictable results on the subgroup model. As TwoStep is a component of the base module of IBM SPSS, it is available in formats that run on the IBM PC, Apple Mac and Linux platforms. Ongoing fee-based support is also available. However, the TwoStep clustering analysis component is not separately available and this software is the most expensive of these three clustering programs, usually involving annual license fees.

In our view, Latent Gold has a steeper learning curve than TwoStep, though the software commands can be menu-driven, there is abundant explanatory material and on-line training courses available, and the results are numerically and graphically presented (including a tri-plot displaying the relationships between subgroups). Latent Gold requires the analyst to determine the optimal subgroup solution but does provide a number of diagnostic measures to inform that decision and clear explanations of the relative merits of those measures. The base version of Latent Gold also allows more complex applications of LCA, such as Latent Class regression modeling and Latent Class multilevel modeling, and can also directly provide model parameters that can be used to classify new individuals who were not in the model building exercise. There is free online support for registered users and the single license fee allows perpetual use of the purchased version. A limitation is that Latent Gold is only available for the IBM PC platform.

SNOB has the steepest learning curve and is completely command line-driven in a Linux shell environment. It is the least user-friendly, requiring input data to be separated into two Linux text files, one containing the data and the other describing the variables, each with a unique syntax. The output needs to be consolidated by extracting information from the Linux shell plus information from a 


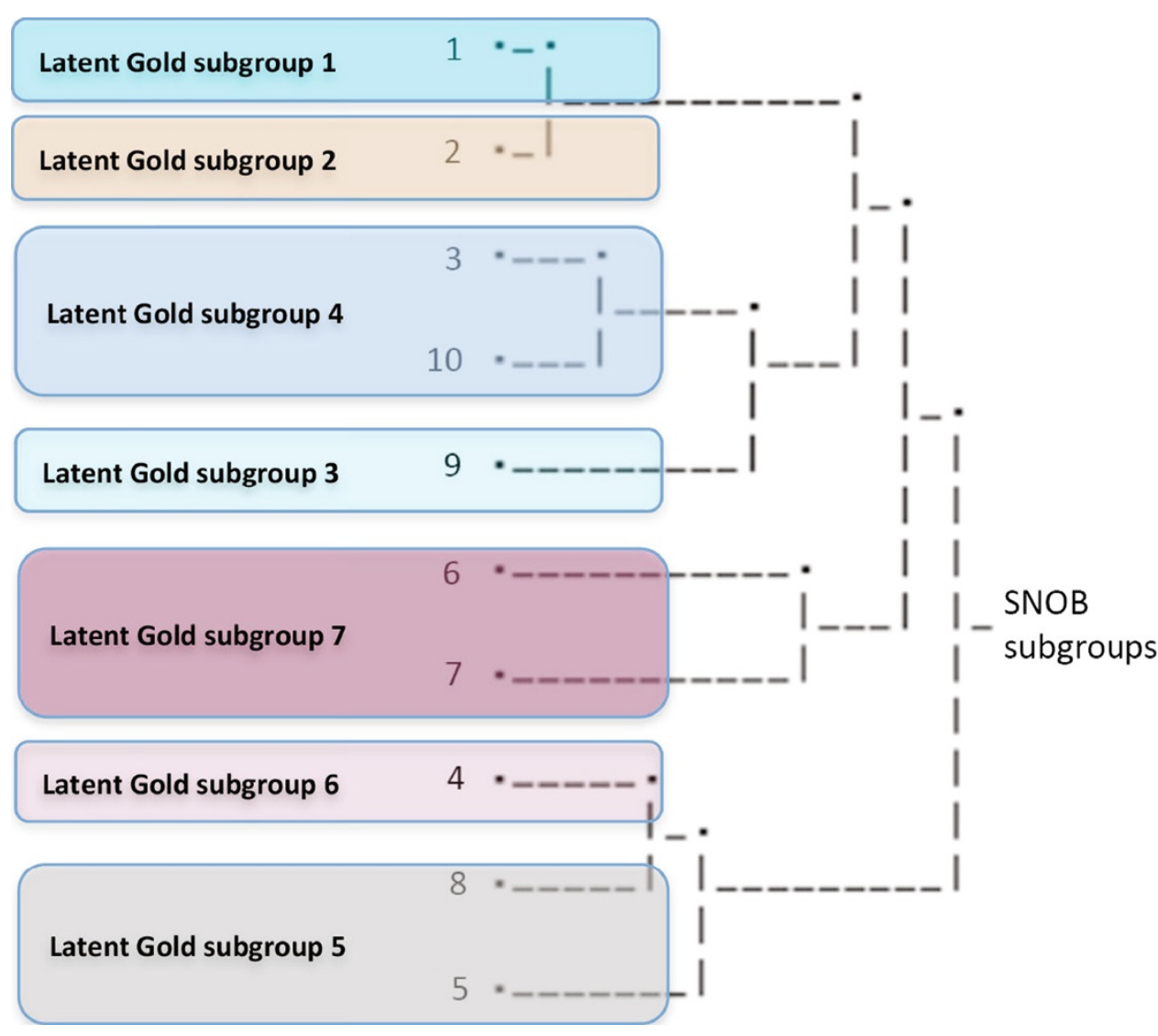

\begin{tabular}{|c|c|c|c|c|c|c|c|c|c|}
\hline \multicolumn{10}{|c|}{ Latent Gold subgroups } \\
\hline & & 1 & \multirow{2}{*}{$\begin{array}{c}2 \\
19\end{array}$} & \multirow{2}{*}{$\begin{array}{c}3 \\
43\end{array}$} & \multirow{2}{*}{$\begin{array}{l}4 \\
7\end{array}$} & \multirow{2}{*}{$\begin{array}{l}5 \\
2\end{array}$} & \multirow{2}{*}{$\begin{array}{l}6 \\
1\end{array}$} & \multicolumn{2}{|l|}{7} \\
\hline \multirow{10}{*}{$\begin{array}{c}\text { SNOB } \\
\text { subgroups }\end{array}$} & 1 & 1,121 & & & & & & 2 & 1,195 \\
\hline & 2 & 15 & 234 & 55 & 13 & 1 & 1 & 1 & 320 \\
\hline & 3 & 1 & 3 & 2 & 79 & 3 & 1 & 2 & 91 \\
\hline & 4 & 1 & 0 & 1 & 0 & 0 & 53 & 0 & 55 \\
\hline & 5 & 0 & 0 & 2 & 0 & 15 & 1 & 0 & 18 \\
\hline & 6 & 1 & 0 & 0 & 0 & 0 & 0 & 32 & 33 \\
\hline & 7 & 2 & 0 & 6 & 0 & 0 & 0 & 8 & 16 \\
\hline & 8 & 0 & 0 & 0 & 0 & 87 & 30 & 0 & 117 \\
\hline & 9 & 1 & 0 & 44 & 6 & 0 & 0 & 0 & 51 \\
\hline & 10 & 3 & 0 & 26 & 43 & 1 & 0 & 1 & 74 \\
\hline & & 1,145 & 256 & 179 & 148 & 109 & 87 & 46 & 1,970 \\
\hline
\end{tabular}

Misclassification $=254 / 1970=12.9 \%$ 
Table 3 Classification performance with real datasets

\begin{tabular}{|c|c|c|c|}
\hline & TwoStep & Latent Gold & SNOB \\
\hline \multicolumn{4}{|l|}{ Number of subgroups detected } \\
\hline $\mathrm{MRI}^{1}$ dataset & 2 & 7 & 10 \\
\hline$\left.M R\right|^{2}$ dataset & 3 & 11 & 15 \\
\hline $\mathrm{MRI}^{3}$ dataset & 2 & 6 & 7 \\
\hline SMS dataset & 2 & 10 & 37 \\
\hline Clinical dataset & Not available & 8 & 9 \\
\hline \multicolumn{4}{|l|}{ Certainty (mean classification probability of disc levels or patients) } \\
\hline $\mathrm{MRI}^{1}$ dataset & Not available & $91.2 \%(S D 11.9 \%)$ & $91.5 \%(11.6 \%)$ \\
\hline$M R I^{2}$ dataset & Not available & $98.9 \%(\mathrm{SD} 3.9 \%)$ & $97.1 \%(\mathrm{SD} 6.6 \%)$ \\
\hline$M R I^{3}$ dataset & Not available & $85.7 \%(S D 19.5 \%)$ & $91.0 \%(\mathrm{SD} 12.7 \%)$ \\
\hline SMS dataset & Not available & $96.5 \%(\mathrm{SD} 8.8 \%)$ & $98.2 \%(S D 4.7 \%)$ \\
\hline Clinical dataset & Not available & $91.4 \%$ (SD12.9\%) & $89.9 \%(\mathrm{SD} 13.5 \%)$ \\
\hline \multicolumn{4}{|l|}{$\begin{array}{l}\text { Reproducibility (10 iterations of each dataset, with identical results } \\
\text { across all datasets) }\end{array}$} \\
\hline Number of subgroups & $100 \%$ agreement & With fixed seed point $=100 \%$ agreement & $100 \%$ agreement \\
\hline $\begin{array}{l}\text { Classification stability (reproducibility of individual disc-levels or } \\
\text { people being classified into each subgroup) }\end{array}$ & $100 \%$ agreement & With fixed seed point $=100 \%$ agreement & $100 \%$ agreement \\
\hline $\begin{array}{l}\text { Classification certainty (reproducibility of the classification probability } \\
\text { of disc levels or patients) }\end{array}$ & Not available & With fixed seed point $=100 \%$ agreement & $100 \%$ agreement \\
\hline
\end{tabular}

report file. The output is mostly numeric, although a tree diagram is produced showing the relationship between 'mother' and 'daughter' subgroups. Some explanatory material is available. This LCA program is free for not-forprofit, academic research but with minimal user support. A factor analytic version of SNOB is also available.

\section{Discussion}

The aim of this study was to perform, using a variety of types of clinical and artificial datasets, a head-to-head comparison of three commonly available clustering methods (TwoStep, Latent Gold and SNOB). Using real clinical datasets, we found that the number of subgroups

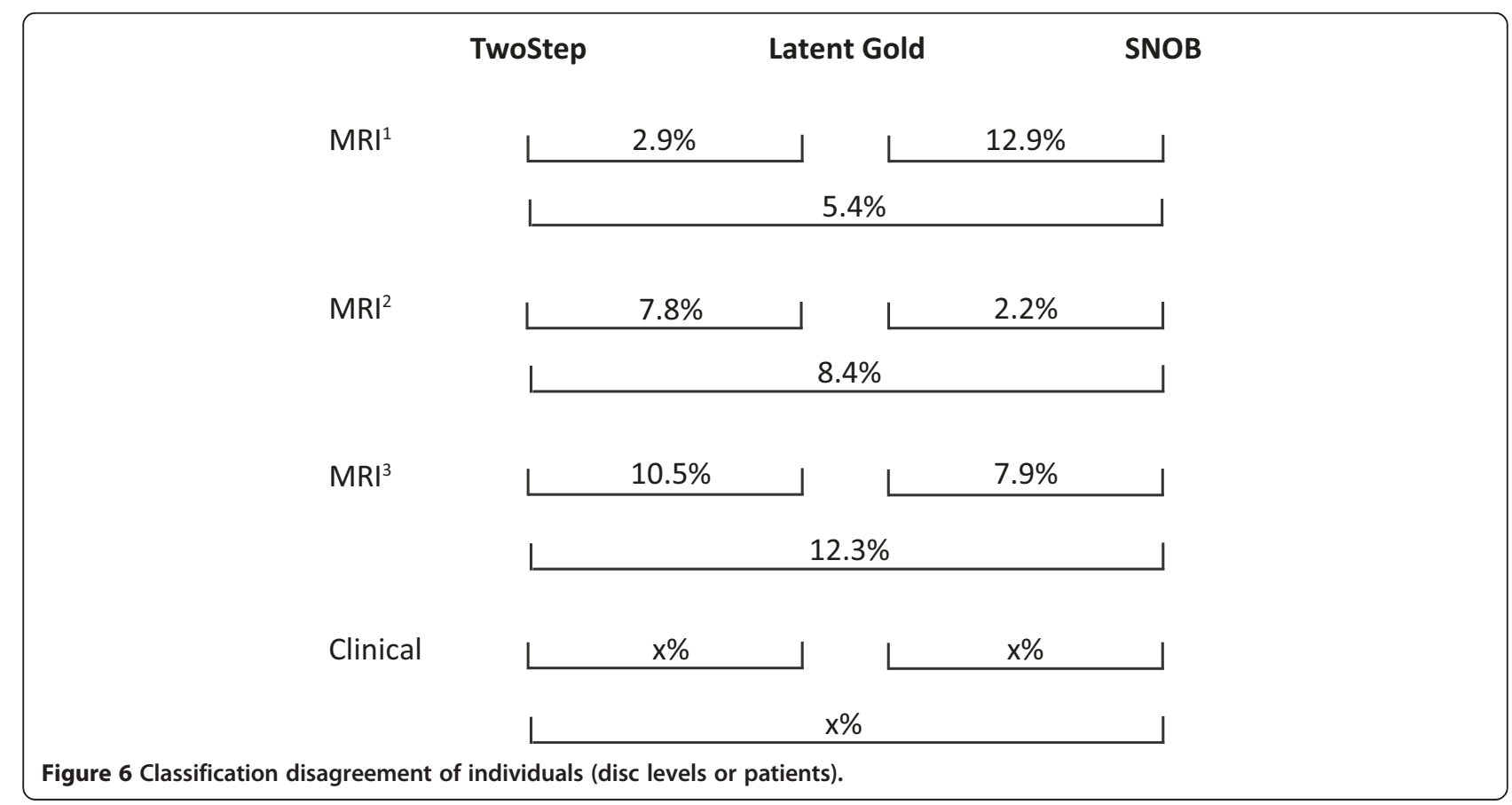


Table 4 Classification performance with artificial datasets

\begin{tabular}{|c|c|c|c|c|c|c|}
\hline \multirow[b]{2}{*}{ Dataset } & \multicolumn{3}{|c|}{ Number of subgroups detected } & \multicolumn{3}{|c|}{ Accuracy of classifying 1000 individuals into subgroups } \\
\hline & TwoStep & Latent Gold & SNOB & TwoStep & Latent Gold & SNOB \\
\hline A1 (3 subgroups) & 3 & 3 & 3 & $100 \%$ & $100 \%$ & $100 \%$ \\
\hline A2 (3 subgroups) & 3 & 3 & 3 & $99.9 \%$ & $99.8 \%$ & $99.9 \%$ \\
\hline A3 (6 subgroups) & 6 & 7 & 6 & $98.7 \%$ & $100 \%$ & $98.4 \%$ \\
\hline A4 (3 subgroups) & 3 & 3 & 3 & $99.4 \%$ & $99.2 \%$ & $99.4 \%$ \\
\hline
\end{tabular}

detected varied, the certainty of classifying individuals into those subgroups varied to some extent, that the findings had perfect reproducibility, that some computer programs were easier to use and that the interpretability of the presentation of findings also varied across programs. With the artificial datasets, all three clustering methods showed a near-perfect ability to detect known subgroups and correctly classify individuals into those subgroups. We believe this information will be useful to clinical researchers.

The number of subgroups detected in all the real datasets varied in a consistent pattern, with TwoStep detecting the least number of subgroups, Latent Gold detecting more subgroups and SNOB detecting the most. This variability in their sensitivity to scoring patterns within the same dataset is problematic, and in the absence of an external reference standard, it is not possible to determine what degree of sensitivity is optimal. To some extent, each clustering method may simply be reflecting the same underlying scoring structure of the data but at different levels of detail. This view appears to be supported by the results from the artificial datasets, which showed nearperfect identification of known subgroups but one instance of a subgroup being split into 'daughter' subgroups. Therefore, the analyst may need to choose a clustering method whose sensitivity level is appropriate for their data and the number of subgroups that are manageable and clinically meaningful. On the other hand, with all subgroup structures, it is possible to collapse subgroups together where, from a clinical perspective, there is good reason to consider them as one, or if the prevalence of a subgroup is so low as to deem it better merged with another. Therefore, an 'overly-sensitive' subgroup structure can be reduced by collapsing 'daughter' subgroups.

Eshghi et al. [29] and Gelbard et al. [13] also showed a lack of consistency across clustering techniques in the number of subgroups detected in real datasets in a comparison of diverse clustering techniques. Eshghi et al. attempted to address this lack of consistency by comparing the subgroup solutions of different clustering programs using measures of within-subgroup homogeneity and between-subgroup heterogeneity to indicate which solutions had better discrimination between subgroups. For example, to determine the within-subgroup homogeneity, they used the sum of squared deviations from the mean to compute the variation when averaged by the number of variables. Although the notion of such an external measure of discrimination is appealing, it may not be helpful in the current context. That is because clinical data is often not normally distributed, especially data collected on something other than an interval scale, and a strength of LCA techniques is their ability to model other types of data and model the probability distributions inherent in each dataset. Therefore, there is no readily apparent external reference standard by which to determine which LCA program results in the optimal subgroup solution in data when subgroup membership is unknown a priori, which is usually the case in clinical research.

Of the datasets that we analysed, only the SMS data were longitudinal. These methods of analysis did not include reference to the longitudinal time sequence inherent in these data. There are more sophisticated modeling methods available for clinical or life course trajectories that do include reference to the longitudinal nature of the data, such as latent class growth analysis and latent class growth mixture modeling [30] but comparison with these techniques was beyond the scope of our study.

Similarly, while the classification certainty (probability) varied between Latent Gold and SNOB in some datasets, this result should be interpreted with some caution and as a general guide only. That is because it was not possible to determine how comparable the measures of classification probability were between LCA programs. LCA methods may calculate classification probability using different approaches.

That there was perfect reproducibility of results (number of clusters, allocation of individuals to clusters, classification probability) is reassuring. However, analysts need to remain mindful that this perfect reproducibility is a result of the programs (except for Latent Gold) choosing an arbitrary but fixed seed point to start their analyses, and that random seed points would in some instances result in different solutions when re-running a model.

In summary, our subjective judgement is that Latent Gold offered the best balance of sensitivity to subgroups, ease of use and interpretability (Table 5). This judgement was based on its ability to manage mixed types of data, the interpretability of its findings and performance measures of subgroups, its ability to perform more complex forms of 
Table 5 Overall summary of three clustering techniques

\begin{tabular}{|c|c|c|c|}
\hline & TwoStep & Latent Gold & SNOB \\
\hline Method & $\begin{array}{l}\text { Distance-based, agglomerative } \\
\text { hierarchical cluster analysis }\end{array}$ & $\begin{array}{l}\text { Finite mixture modeling } \\
\text { to probabilistically identify } \\
\text { latent classes }\end{array}$ & $\begin{array}{c}\text { Finite mixture modeling to } \\
\text { probabilistically identify latent classes }\end{array}$ \\
\hline $\begin{array}{l}\text { Stopping rule to identify number of } \\
\text { subgroups }\end{array}$ & $\begin{array}{c}\begin{array}{c}\text { Automated using either 'Bayesian information criterion' or 'Akaike's } \\
\text { information criterion' }\end{array}\end{array}$ & $\begin{array}{l}\text { Analyst choice using various criteria, } \\
\text { including 'Bayesian information } \\
\text { criterion', unexplained variance, } \\
\text { Chi-square p-value }\end{array}$ & $\begin{array}{l}\text { Automated using 'Minimum } \\
\text { message length' principle }\end{array}$ \\
\hline Suitable data types & $\begin{array}{l}\text { Ordinal data require recoding } \\
\text { as dichotomous or handled as } \\
\text { if interval data }\end{array}$ & All types & All types \\
\hline $\begin{array}{l}\text { Report classification probability of } \\
\text { individuals }\end{array}$ & No & Yes & Yes \\
\hline Sensitivity to subgroups & Least & Middle & Most \\
\hline Reproducibility & Very high & Very high & Very high \\
\hline Accuracy & Very high & Very high & Very high \\
\hline Cost & Most expensive & Less expensive & Free \\
\hline Support & $\begin{array}{l}\text { Extensive documentation, fee-based } \\
\text { support available }\end{array}$ & $\begin{array}{l}\text { Extensive documentation and some } \\
\text { free support available }\end{array}$ & $\begin{array}{l}\text { Some documentation but minimal } \\
\text { support available }\end{array}$ \\
\hline $\begin{array}{l}\text { Interpretability of presentation of } \\
\text { results }\end{array}$ & $\begin{array}{l}\text { Results are presented numerically } \\
\text { and graphically (charts of certainty } \\
\text { of the subgroup structure, bar and pie } \\
\text { charts of cluster frequencies, and } \\
\text { charts displaying the importance of } \\
\text { specific variables to subgroups) }\end{array}$ & $\begin{array}{c}\text { Results are presented numerically } \\
\text { and graphically (including a tri-plot } \\
\text { displaying the relationships between subgroups) }\end{array}$ & $\begin{array}{l}\text { Results are mostly numeric (although } \\
\text { a tree diagram is produced showing } \\
\text { the relationship between 'mother' } \\
\text { and 'daughter' subgroups) }\end{array}$ \\
\hline Learning curve (subjective judgement) & Easy & Middle & Hard \\
\hline
\end{tabular}


LCA, its capacity to generate model parameters that can be used to classify new individuals, and the accessibility of support. A further consideration was that while allowing analyst discretion in choosing the optimal subgroup solution might potentially introduce the capacity for bias, this process also makes explicit the criteria that were used in that choice, which may need to differ depending on the characteristics of the data and the clinical question. Compared to TwoStep, we valued the higher sensitivity, much better handling of ordinal and mixed types of data, and the more detailed output of Latent Gold. Whereas the main reason that we preferred Latent Gold over SNOB was its user-friendliness. We recognise that this judgement of the best clustering to use might vary depending on the analyst's own expertise and support, the types of data involved, and the clinical questions to be answered.

A strength of this study is that it was performed using a variety of real and artificial datasets and use of a range of performance criteria. A limitation was that other LCA methods are available that we did not test, as a comprehensive comparison of all available LCA methods was beyond the scope of the study.

\section{Conclusions}

This study compared three clustering methods (SPSS TwoStep, Latent Gold and SNOB) using a variety of datasets and performance criteria. The results from the real datasets indicated that the number of subgroups detected varied, the certainty of classifying individuals into those subgroups varied to some extent, the findings had perfect reproducibility, some programs were easier to use and the interpretability of their presentation of findings also varied across programs. The results from the artificial datasets indicated that all three clustering techniques showed a near-perfect ability to detect known subgroups and correctly classify individuals into those subgroups. Our judgement was that Latent Gold offered the best balance of sensitivity to subgroups, ease of use and interpretability but we recognise that other analysts may reach different conclusions depending on their available level of statistical support, the types of data they work with and the clinical questions they address. We believe this information will be useful to clinical researchers making decisions about which clustering methods might be appropriate to their circumstances.

\section{Abbreviations \\ BIC: Bayesian Information Criterion; GP: General medical Practitioners; LBP: Low Back Pain; LCA: Latent Class Analysis; MRI: Magnetic Resonance Imaging; SMS: Short Message Service (text messaging); TwoStep: TwoStep Cluster Analysis in IBM SPSS.}

\section{Competing interests}

No author has any competing interests. The manuscript submitted does not contain information about medical devices or drugs. No benefits in any form have been, or will be, received from a commercial party related directly or indirectly to the subject of this manuscript. None of the authors have any relationship with the suppliers of the tested software other than as a customer.

\section{Authors' contributions}

PK, RKJ and AK were involved in the design of the study, data analysis, interpretation of data, revision of the manuscript. PK wrote the initial draft of the manuscript. All authors read and approved the final manuscript.

\section{Acknowledgements}

PK and RKJ were partially supported, and AK was fully supported, by grants from the Danish Foundation for the promotion of Chiropractic research and post-graduate education. Thanks to Hanne Albert, Per Kjaer and the 'Backs on Funen' Steering Committee for data access.

\section{Author details}

${ }^{1}$ School of Sports Science and Clinical Biomechanics, University of Southern Denmark, Campusvej 55, Odense M 5230, Denmark. ${ }^{2}$ Research Department, Spine Centre of Southern Denmark, Hospital Lillebaelt, Institute of Regional Health Services Research, University of Southern Denmark, Middelfart, Denmark. ${ }^{3}$ Nordic Institute of Chiropractic and Clinical Biomechanics, University of Southern Denmark, Odense, Denmark.

Received: 6 September 2013 Accepted: 24 September 2014

Published: 2 October 2014

\section{References}

1. Hill JC, Whitehurst DG, Lewis M, Bryan S, Dunn K, Foster NE, Konstantinou K, Main CJ, Mason E, Somerville S, Sowden G, Vohora K, Hay EM: Comparison of stratified primary care management for low back pain with current best practice (STarT Back): a randomised controlled trial. Lancet 2011, 378(9802):1560-1571.

2. Hingorani AD, Windt DA, Riley RD, Abrams K, Moons KG, Steyerberg EW, Schroter S, Sauerbrei W, Altman DG, Hemingway H: Prognosis research strategy (PROGRESS) 4: Stratified medicine research. BMJ 2013, 346:e5793.

3. Lim SS, Vos T, Flaxman AD, Danaei G, Shibuya K, Adair-Rohani H, Amann M, Anderson HR, Andrews KG, Aryee M, Atkinson C, Bacchus LJ, Bahalim AN, Balakrishnan K, Balmes J, Barker-Collo S, Baxter A, Bell ML, Blore JD, Blyth F, Bonner C, Borges G, Bourne R, Boussinesq M, Brauer M, Brooks P, Bruce NG, Brunekreef B, Bryan-Hancock C, Bucello C, et al: A comparative risk assessment of burden of disease and injury attributable to 67 risk factors and risk factor clusters in 21 regions, 1990-2010: a systematic analysis for the Global Burden of Disease Study 2010. Lancet 2012, 380(9859):2224-2260.

4. Jensen RK, Jensen TS, Kjaer P, Kent P: Can pathoanatomical pathways of degeneration in lumbar motion segments be identified by clustering MRI findings. BMC Musculoskelet Disord 2013, 14(1):198.

5. Takatalo J, Karppinen J, Niinimaki J, Taimela S, Mutanen P, Sequeiros RB, Nayha S, Jarvelin MR, Kyllonen E, Tervonen O: Association of modic changes, Schmorl's nodes, spondylolytic defects, high-intensity zone lesions, disc herniations, and radial tears with low back symptom severity among young Finnish adults. Spine 2012, 37(14):1231-1239.

6. Barban N, Billari FC: Classifying life course trajectories: a comparison of latent class and sequence analysis. J R Stat Soc 2012, 61(5):765-784.

7. Axen I, Bodin L, Bergstrom G, Halasz L, Lange F, Lovgren PW, Rosenbaum A, Leboeuf-Yde $C$, Jensen I: Clustering patients on the basis of their individual course of low back pain over a six month period. $B M C$ Musculoskelet Disord 2011, 12:99.

8. Kent P, Keating JL, Leboeuf-Yde C: Research methods for subgrouping low back pain. BMC Med Res Methodol 2010, 10:62. doi:10.1186/1471-2288-10-62.

9. Klebanoff MA: Subgroup analysis in obstetrics clinical trials. Am J Obstet Gynecol 2007, 197:119-122.

10. Flynn T, Fritz JW, Whitman M, Wainner RS, Magel J, Rendeiro D, Butler B, Garber M, Allison S: A clinical prediction rule for classifying patients with low back pain who demonstrate short-term improvement with spinal manipulation. Spine 2002, 27(24):2835-2843.

11. Beneciuk JM, Robinson ME, George SZ: Low back pain subgroups using fear-avoidance model measures: results of a cluster analysis. Clin J Pain 2012, 28(8):658-666.

12. Bacher J, Wenzig K, Vogler M: SPSS TwoStep Cluster - a first evaluation. In Work and discussion paper. Erlangen-Nuremberg, Germany: Department of Sociology, Social Science Institute, Friedrich-Alexander-University; 2004:1-30. 
13. Gelbard R, Goldman O, Spiegler I: Investigating diversity of clustering methods: An empirical comparison. Data Knowl Eng 2007, 63:155-166.

14. Magidson J, Vermunt JK: Latent class models for clustering: A comparison with k-means. Can J Market Res 2002, 20:1-9.

15. Haughton D, Legrand $P$, Woolford S: Review of three Latent Class Cluster Analysis packages: Latent GOLD, poLCA, and MCLUST. Am Stat 2009, 63(1):81-91.

16. SPSS: SPSS Base 17.0 Users guide. Chicago, IL, USA: SPSS Inc; 2009

17. Vermunt JK, Magidson J: Latent Gold 4.0 users's guide. Belmont, Massachusetts, USA: Statistical Innovations Inc; 2005.

18. Wallace CS: Statistical and inductive inference by minimum message length New York, USA: Springer; 2005.

19. Wallace CS, Boulton DM: An information measure for classification. Comput J 1968, 11(2):185-194.

20. Wallace CS, Dowe DL: MML clustering of multi-state, Poisson, von Mises circular and Gaussian distributions. Stat Comput 2000, 10(1):73-83.

21. Kjaer P, Korsholm L, Bendix T, Sorensen JS, Leboeuf-Yde C: Modic changes and their associations with clinical findings. Eur Spine J 2006, 15:1312-1319.

22. Jensen TS, Sorensen JS, Kjaer P: Intra- and interobserver reproducibility of vertebral endplate signal (modic) changes in the lumbar spine: The Nordic modic consensus group classification. Acta Radiol 2007, 48:748-754

23. Jensen RK, Leboeuf-Yde C, Wedderkopp N, Sorensen JS, Manniche C: Rest versus exercise as treatment for patients with low back pain and Modic changes. A randomized controlled clinical trial. BMC Med 2012, 10:22.

24. Albert HB, Briggs AM, Kent P, Byrhagen A, Hansen C, Kjaergaard K: The prevalence of MRI-defined spinal pathoanatomies and their association with modic changes in individuals seeking care for low back pain. Eur Spine J 2011, 20(8):1355-1362.

25. Kent P, Briggs AM, Albert HB, Byrhagen A, Hansen C, Kjaergaard K, Jensen TS: Inexperienced clinicians can extract pathoanatomic information from MRI narrative reports with high reproducibility for use in research/ quality assurance. Chiropr Man Therap 2011, 19(1):16.

26. Eirikstoft $H$, Kongsted A: Patient characteristics in low back pain subgroups based on an existing classification system. A descriptive cohort study in chiropractic practice. Man Ther 2014, 19(1):65-71.

27. Kent $P$, Kongsted $A$ : Identifying clinical course patterns in SMS data using cluster analysis. Chiropr Man Therap 2012, 20(1):20.

28. Kongsted A, Johannesen E, Leboeuf-Yde C: Feasibility of the STarT back screening tool in chiropractic clinics: a cross-sectional study of patients with low back pain. Chiropr Man Therap 2011, 19:10

29. Eshghi A, Haughton D, Legrand P, Skaletsky M, Woolford S: Identifying groups: A comparison of methodologies. J Data Sci 2011, 9:271-291.

30. Twisk J, Hoekstra T: Classifying developmental trajectories over time should be done with great caution: a comparison between methods. J Clin Epidemiol 2012, 65(10):1078-1087.

doi:10.1186/1471-2288-14-113

Cite this article as: Kent et al: A comparison of three clustering methods for finding subgroups in MRI, SMS or clinical data: SPSS TwoStep Cluster analysis, Latent Gold and SNOB. BMC Medical Research Methodology 2014 14:113.

\section{Submit your next manuscript to BioMed Central and take full advantage of:}

- Convenient online submission

- Thorough peer review

- No space constraints or color figure charges

- Immediate publication on acceptance

- Inclusion in PubMed, CAS, Scopus and Google Scholar

- Research which is freely available for redistribution 